\title{
A review and analysis of strategies for prediction, prevention and management of post-operative atrial fibrillation after non-cardiac thoracic surgery
}

\author{
Heather Smith ${ }^{1}$, Ching Yeung ${ }^{2}$, Stephen Gowing ${ }^{2}$, Mouhannad Sadek ${ }^{3}$, Donna Maziak ${ }^{2}$, Sebastien \\ Gilbert $^{2}$, Farid Shamji ${ }^{2}$, Patrick Villeneuve ${ }^{2}$, Sudhir Sundaresan ${ }^{2}$, Andrew Seely ${ }^{2}$ \\ ${ }^{1}$ Division of General Surgery, ${ }^{2}$ Division of Thoracic Surgery, ${ }^{3}$ Division of Cardiology, The Ottawa Hospital, University of Ottawa, Ottawa, ON, \\ Canada \\ Contributions: (I) Conception and design: H Smith, A Seely; (II) Administrative support: H Smith; (III) Provision of study materials or patients: None; \\ (IV) Collection and assembly of data: H Smith, A Seely, M Sadek, F Shamji, D Maziak, R Sundaresan; (V) Data analysis and interpretation: H Smith, \\ A Seely, M Sadek, F Shamji, D Maziak, R Sundaresan; (VI) Manuscript writing: All authors; (VII) Final approval of manuscript: All authors. \\ Correspondence to: Heather Smith. Department of Surgery, Division of General Surgery, The Ottawa Hospital, 501 Smyth Rd, Ottawa, ON K1H 8L6, \\ Canada. Email: hesmith@toh.ca.
}

\begin{abstract}
Atrial fibrillation (AF) is the most common sustained arrhythmia after non-cardiac thoracic surgery and is associated with a significant increase in perioperative morbidity, intensive care unit (ICU) admission, and mortality. Practical guidance is needed to assist clinicians in managing this critical issue and direct further research. Here we aim to provide a synoptic review and analysis of the literature to distil practical recommendations for prediction, prevention and management of post-operative atrial fibrillation (POAF) suitable for clinical application and further evaluation. To predict POAF, risk factors including age, gender, elevated pre-operative heart rate and extent of surgical resection have been reproducibly identified and integrated into scoring systems. To prevent POAF, prophylactic therapy with beta-blockers, amiodarone, or magnesium have demonstrated to be effective, but need further trials in high-risk populations. To manage unstable POAF that precipitates hypotension and hypoperfusion, although rare, requires immediate electrocardioversion to restore cardiac output and adequate oxygen delivery. For hemodynamically stable patients, rate control and prevention of adverse events are the objectives. We propose an individualized approach aimed at rate control using initial incremental low dose beta-blocker or calcium channel blocker (CCB) therapy with close monitoring of a patient's response, and continuation of the drug that they respond to, along with simultaneous identification and reduction of triggers of $\mathrm{AF}$, in order for spontaneous return to sinus rhythm. For patients who persistently fail to respond to rate control therapy, rhythm control may be considered using an agent selected based on the patient's comorbidities and the medications' side effect profile. While controversial and requiring further study, anticoagulation therapy is recommended in patients with risk factors for thromboembolic events after 48 hours of persistent AF. We recommend continuous prospective monitoring of incidence and severity of POAF to track the impact of protocols to predict, prevent and manage POAF.
\end{abstract}

Keywords: Post-operative atrial fibrillation (POAF); thoracic surgery; prediction; prevention; management

Submitted Jul 09, 2018. Accepted for publication Sep 27, 2018.

doi: $10.21037 /$ jtd.2018.09.144

View this article at: http://dx.doi.org/10.21037/jtd.2018.09.144 


\section{Introduction}

Atrial fibrillation (AF) is the most commonly sustained arrhythmia after non-cardiac thoracic surgery, occurring after $12-44 \%$ of pulmonary and esophageal resections (1). It is associated with a significant increase in post-operative morbidity, length of stay (LOS), intensive care unit (ICU) admission and mortality $(1,2)$. Patients who develop postoperative atrial fibrillation (POAF) often experience an increased LOS of 2 to 14 days (3). If sustained, POAF can increase a patient's risk of thromboembolic events and it an independent risk factor of stroke in the 30 days following development of new sustained AF $(4,5)$. Therefore, in some cases anticoagulation is critical (5). AF is associated with increased mortality risk after esophagectomy (mortality increase from $4.8 \%$ to $8.1 \%, \mathrm{P}=0.04$ ) (6) and decreased long-term survival after lobectomy (HR 3.75; 95\% CI, 1.44 to 9.08 ) (1). As AF is both common and impactful, efforts to effectively and optimally predict, prevent and manage POAF is critical to improving quality of thoracic surgical care.

Despite significant developments in our understanding of the pathogenesis of $\mathrm{AF}$ and risk factors, there is no practical synthesis regarding the prediction, prevention and management of POAF. Multiple prevention therapies have been proven effective at reducing the incidence of POAF, however due to the dearth of trials and concern regarding adverse effects, prophylaxis has not been recommended $(7,8)$. The American Association of Thoracic Surgeons (AATS) recently published practice guidelines for the prophylaxis and management of POAF (8). Our aim here is not to reproduce these practice guidelines; rather, the aim of this review and analysis is to provide a practical, feasible and clinically applicable strategy for the prediction, prevention and management of POAF in patients undergoing noncardiac thoracic surgery. The following review, analysis and recommendations are designed to assist with clinical application in thoracic surgery centers, along with tracking of the incidence and severity of POAF to evaluate its clinical impact.

\section{Methods}

We searched Medline, the Cochrane central Registry of Randomized Controlled Trials, Ecerpta of Medica Database (EMBASE) National Guidelines Clearinghouse, Canadian Medical Association (CMA) Infobase, National Health Service (NHS) Evidence, Scotland Intercollegiate
Guideline Network (SIGN) and National Institute for Health and Care Excellence (NICE) guidelines for articles published after 2005 using several searches specific to topics within this paper. For prediction and prophylaxis we used the terms AF, thoracic surgery, POAF, predict, prevent, and prophylaxis. For management we used the search terms $\mathrm{AF}$, thoracic surgery, guidelines and consensus. To further assess rate control therapy, we did additional searches adding the terms diltiazem, calcium channel blocker (CCB), metoprolol and beta blocker. Further searching for articles citing relevant studies and bibliographies of useful articles was done to expand our search. All authors provided iterative review of the results, discussing and finalizing recommendations. Our recommendations are intended to be useful in so far as they reflect a practical and feasible approach to systematically address the problem of POAF, including rigorously evaluating the impact of this approach. It is important to note that the recommendations are not guidelines, and the search methodology for articles was not that of a systematic review, and we therefore acknowledge there may be missing relevant and important citations.

\section{Prediction}

The mechanisms of POAF remain complex and multifactorial. Since AF was first documented using the electrocardiogram in 1909 by Sir Thomas Lewis (9), it has been the subject of intense scrutiny. Numerous factors underlying the pathogenesis of AF have been identified, including genetics involving $\mathrm{K}+$ and $\mathrm{Na}+$ channels, increased atrial pressure (e.g., due to essential hypertension, valvular disease, pulmonary hypertension, and obstructive sleep apnea), inflammation and altered autonomic modulation (e.g., increased sympathetic tone) (10). POAF is thought to have similar pathogenic mechanisms, with some differences. After cardiothoracic surgery, the incidence of POAF is highest on postoperative days 2, closely tied to the highest levels of reactive oxidant species and inflammatory cytokines, including C-reactive protein, highlighting the importance of inflammation $(11,12)$. While a full discussion of the multifactorial mechanisms of POAF is beyond the scope of this review, what appears consistent is the concept that a variety of factors predispose the heart to be at increased risk for $\mathrm{AF}$, and surgery (specifically cardiac or thoracic surgery), may elevate a patient over their AF threshold, leading to POAF (13).

Multiple patient and surgical factors have been identified 
Table 1 statistically significant independent risk factors for POAF in thoracic surgery

\begin{tabular}{|c|c|c|c|c|c|c|c|c|c|}
\hline Publication & M & No POAF (total) & $\uparrow$ Age & $\uparrow$ Resection & Male gender & $\uparrow$ Disease & $\uparrow \mathrm{BNP}$ & $\uparrow$ Resting HR & $\mathrm{Hx}$ of $\mathrm{VD}$ \\
\hline \multicolumn{10}{|l|}{ Pulmonary resection } \\
\hline $\begin{array}{l}\text { Onaitis et al., } \\
2010 \text { (14) }\end{array}$ & $\sqrt{ }$ & $65[415]$ & $>67$ & $\sqrt{ }$ & $\sqrt{ }$ & $\sqrt{ }$ & & & \\
\hline $\begin{array}{l}\text { Kotova et al., } \\
2017 \text { (15) }\end{array}$ & & 113 [933] & $>75$ & & & & & & $\sqrt{ }$ \\
\hline $\begin{array}{l}\text { Cardinale et al., } \\
2007 \text { (16) }\end{array}$ & $\sqrt{ }$ & 441 & & & & & $\sqrt{ }$ & & $\sqrt{ }$ \\
\hline $\begin{array}{l}\text { Imperatori et al., } \\
2012 \text { (1) }\end{array}$ & $\sqrt{ }$ & $45[454]$ & & & & & & & \\
\hline \multicolumn{10}{|l|}{ Esophageal resection } \\
\hline Rao et al., 2012 (6) & & 209 [997] & $>65$ & & $\sqrt{ }$ & $\sqrt{ }$ & & & \\
\hline \multicolumn{10}{|c|}{ All non-cardiac thoracic surgery (pulmonary and esophageal) } \\
\hline $\begin{array}{l}\text { Simmers et al., } \\
2015 \text { (17) }\end{array}$ & & 108 [742] & & & & & $\sqrt{ }$ & & \\
\hline $\begin{array}{l}\text { Amar et al., } \\
2012 \text { (18) }\end{array}$ & $\sqrt{ }$ & 65 [454] & $>65$ & & $\sqrt{ }$ & & $\sqrt{ }$ & & \\
\hline $\begin{array}{l}\text { Passman et al., } \\
2005 \text { (19) }\end{array}$ & & 147 [856] & $>55$ & & $\sqrt{ }$ & & & $\sqrt{ }$ & \\
\hline $\begin{array}{l}\text { Vaporciyan et al., } \\
2004 \text { (20) }\end{array}$ & & $319[2,558]$ & $>50$ & $\sqrt{ }$ & & & & & \\
\hline
\end{tabular}

$\uparrow$, indicates increase. M, study selected for patients with malignant disease only; No POAF (total), number of individuals who develop post-operative atrial fibrillation; BNP, b-type natriuretic peptide; HR, pre-operative heart rate at rest; Hx of VD, history of vascular disease.

as independent predictors of POAF, outlined in Table 1. Patient factors identified as independent predictors of POAF include advanced age, a history of congestive heart failure, concomitant lung disease, preoperative tachycardia, neoadjuvant chemotherapy and male gender (1,2,6,15-21). Increasing age is a factor that has consistently demonstrated elevated risk of $\mathrm{AF}$, and lead to its sustained presence. This may be due to the age-associated myocardial apoptosis and fibrosis contributing to the intra-atrial conduction delay, and ultimately reentrant circuits responsible for AF (22). Male gender has been shown to be linked with increased risk for $\mathrm{AF}$, which may be due to general increased atrial size or the increased proinflammatory immune response seen in male patients compared with females $(19,22)$. As evident in the cardiac surgery literature, surgical stress and burden of disease has also been shown to play a significant role in the development of AF after thoracic surgery. For instance, patients undergoing bilobectomy or pneumonectomy have been found to have significantly increased risk of AF over patients undergoing a lobectomy (odds ratio of $1.64 \mathrm{vs} .1 .95$ respectively) (14). Elevated brain natriuretic peptide (BNP) level has also been demonstrated to be an independent predictor of AF. This may be due to its concomitant elevation in patients with advanced age, lung disease and coronary artery disease which are independent risk factors for AF $(17,20)$. Multiple small heterogenous studies have demonstrated association of BNP with POAF, however an appropriate threshold has yet to be defined. Independent risk factors have been combined by Passman and colleagues to form a prediction model that can be easily applied by clinicians in the preoperative setting based on a patient's age, gender and resting heart rate and overall classification accuracy of $67 \%$ (c-statistic $0.65-0.73$ ) (19). The prediction rule should be externally validated to assist with guiding prophylactic therapy in high-risk patients.

\section{Recommendation}

Multiple existing predictive models are capable of identifying patients at increased risk for POAF; however, 
clinical application of these risk models first requires external validation, followed by ongoing evaluation and improvement over time.

\section{Prevention}

To prevent POAF, multiple pharmacologic agents have been investigated with varying levels of effectiveness and risk. Trials directly comparing medical therapy agents are limited and heterogeneous, and much of the evidence is extrapolated from cardiac surgery trials. A recent metaanalysis found that pharmacologic prophylaxis including beta-blockers, amiodarone, and magnesium, significantly reduced the risk of POAF and major adverse cardiovascular events over standard care (odds ratio of 0.33 ; 95\% CI, $0.22-0.49$ and $0.39 ; 95 \%$ CI, 0.17-0.87) (23). Among these agents, beta-blockers demonstrated the greatest effectiveness at preventing AF (reduction from $40 \%$ to 9\%) (23) but have not been recommended as prophylaxis in the 2014 AATS guidelines due to concern of bronchospasm and hypotension developing in patients undergoing lung resection (8). Although the issue of bronchospasm may be overstated, the issue of bradycardia, hypotension, stroke (increase of 98\%), and mortality [increase of $27 \%$ identified in patients starting beta-blocker for myocardial infarction (MI) risk reduction pre-operatively] (24) is of major concern. The evidence for preoperative initiation of beta-blockade in patients at risk of POAF is conflicting and further evaluation is needed (21). For patients already on beta-blocker therapy, the general recommendation is to continue therapy (8). Amiodarone has also been shown in multiple RCTs to significantly reduce $\mathrm{AF}$ (from $20.5 \%$ to $10 \%$ ) (25). Initially, amiodarone was considered unsafe in thoracic surgery after a small study in 1994 demonstrated an association between the use of amiodarone and acute respiratory distress syndrome (ARDS) after pneumonectomy (26). However, multiple studies have since demonstrated that low doses of amiodarone are safe and effective in patients with chronic lung disease $(27,28)$. Magnesium has also been demonstrated to be effective in reducing the risk of AF. In a trial comparing its use with amiodarone $v s$. control, it was found to be inferior to amiodarone but better tolerated (with an incidence of POAF of $20.5 \%$ in the control group, $10 \%$ in the amiodarone group, and $12.5 \%$ in the magnesium group) (25). CCBs have been less effective on average for prophylaxis than the other above mentioned therapies and are associated with risk of bradycardia and hypotension, and as with beta blockers, must be used with caution in patients with impaired systolic function. Thus, prophylactic therapy with beta-blocker agents, amiodarone and magnesium has the potential to reduce AF in high-risk patients, but is rarely implemented in clinical practice (23). To date there is inadequate data to provide clear recommendations for prophylaxis in all patients. Further evaluation of prophylactic therapy in high-risk patients is needed to understand this potentially impactful aspect of managing POAF.

\section{Recommendations}

Prophylactic therapy with beta-blocker agents, amiodarone, $\mathrm{CCB}$ and magnesium may be considered to reduce the risk of POAF; however providing prophylaxis to all patients is not recommended because the exposure of all patients to the risk of adverse events outweighs the benefit experienced by only a few patients in preventing POAF. The approach of implementing prophylaxis only in high-risk patients only to reduce incidence and severity of POAF is promising yet unproven, and requires further study to evaluate safety and effectiveness.

\section{Management}

The objective of POAF management is to restore cardiac output and ensure adequate oxygen delivery. If the patient is hemodynamically unstable, electrocardioversion is first-line therapy to convert the patient back to a sinus rhythm. If cardioversion is performed 48 hours after sustained $\mathrm{AF}$, echocardiogram is recommended to detect an atrial thrombus (8). However, the vast majority of patients are hemodynamically stable or have mild alteration in blood pressure. In these patients, rate control remains the principal objective of initial therapy to allow for adequate ventricular filling, restoration of cardiac output and reduce the morbidity and cardiomyopathy associated with tachycardia (29). Concurrently with rate control, triggers of $\mathrm{AF}$ need to be addressed to generate to optimal conditions for the patients to spontaneously and safely return to a sinus rhythm.

As rate control is the primary goal of initial treatment, numerous pharmacologic agents have been investigated to manage POAF-associated tachycardia in hemodynamically stable patients. Rhythm control is not recommended as initial treatment because the complex side effect profile, associated toxicity and the frequent resolution of POAF within 48 hours precludes their use (30). 
Rate control may be achieved with intravenous betablocker, and/or CCB therapy, both considered safe first-line agents. Multiple trials performed in the 1990s demonstrated their effectiveness at rate control (29). However, the literature comparing the two drug classes is sparse. We were only able to identify two small randomized trials comparing beta-blockers with $\mathrm{CCB}$ in $\operatorname{POAF}(31,32)$. The first study included 64 patients admitted to intensive care who developed post-operative supraventricular tachycardia. Patients were randomized to receive esmolol or diltiazem. In the sub group analysis of patients with POAF, conversion to sinus rhythm at 2 hours was seen more readily with beta-blockers than CCB however this was not statistically significant $(59 \%$ vs. $27 \%, \mathrm{P}=0.067)$. Overall both agents were effective at achieving rate control in majority of patients by 12 hours with no significant difference in hospital LOS or mortality (31). A second trial of 60 patients who developed POAF after coronary artery bypass, were randomized to receive treatment with esmolol or diltiazem. They found that esmolol was associated with greater conversion to sinus rhythm at $<6$ hours $(67 \% v s$. $13 \%, \mathrm{P}=0.03)$ and diltiazem was associated with greater rate control at $<12$ hours ( $100 \%$ vs. $40 \%, \mathrm{P}=0.045)$ with no significant difference in adverse events between the two groups (32). These findings are also consistent with the few small trials conducted with patients presenting with new $\mathrm{AF}$ to the ED (Emergency Department), but without the distinction of beta-blockers resulting in earlier conversion to sinus $(33,34)$. The difference in conversion with betablockers post-operatively may be due to the heightened adrenergic tone seen in the post-operative state. Based on our review, there is limited difference between agents in achieving rate control. Metoprolol is more readily available due to its ability to be stored at room temperature thus we recommend it as initial therapy. Although similar in response, we noted that the dose required to achieve rate control with both agents varies between patients.

Individualized titrated dosing to achieve rate control is necessary, and variable response is observed. In the majority of the trials reviewed, the dose to achieve rate control varied and was often titrated by clinicians based on patient response (35). In the AFFIRM trial, rate control agents were adjusted to achieve pre-selected rate control guidelines, which included resting heart rate of $<80$ and 6 min walk test heart rate $<110$ (36). In a recent trial for acute management of AF in ICU patients after non-cardiothoracic surgery, patients received titrated doses of either metoprolol or diltiazem to achieve a heart rate of $<110$. Those receiving metoprolol required doses ranging between 5-15 $\mathrm{mg}$ and those receiving diltiazem received an initial bolus of $10-46 \mathrm{mg}$ with $45 \%$ of patients requiring a repeat bolus (30). The variability in dosing required for treatment effect undermines direct comparison of agents with RCTs and emphasizes the need to develop and evaluate more individualized strategies to treating POAF that incorporate continuous monitoring, titration of dosing to patient response and advocates for multi-agent therapy. Figure 1 outlines such an approach. Using the most recent evidence on pharmacotherapy for rate control as well as triggers of $\mathrm{AF}$ this algorithm facilitates a comprehensive approach to individualized therapy. In this algorithm, low doses of first line rate control therapies are administered until heart rate is $<110 \mathrm{bpm}$ (the heart rate demonstrated to be effective at improving outcomes for patients with AF by the RACE II trial) (26). The addition of digoxin has been shown to improve rate control and can be added if inadequate response to initial therapy (as demonstrated by the AFFIRM trial: $75 \%$ vs. $68 \%$ when added to beta-blocker, $66 \%$ vs. $60 \%$ for CCB) (36). In patients with decompensated heart failure or ejection fraction $<35 \%$, beta-blocker and CCB therapy should be avoided for their negative inotropic effect and amiodarone should be given instead (29). Patients will have inconsistent response to therapy and therefore require an individualized approach with continuous monitoring with repeat dosing/addition of another pharmacological agents according patient's response. As previously discussed, there are multiple factors contributing to a patient's risk of POAF and simultaneous attempts should be made to reverse them.

Operative dissection and inflammatory changes may be sufficient to provoke AF, however, other reversible conditions may alter the patient's adrenergic response and conversion to $\mathrm{AF}(37,38)$. A suggested approach to initial assessment and investigations to identify triggers such as pneumothorax, pulmonary embolism, infection (including pneumonia, empyema or anastomotic leak), bleeding or electrolytes disturbances is outlined in Figure 1. Addressing reversible triggers may contribute to earlier return to sinus rhythm. A comprehensive approach to AF with individualized rate control therapy and reduction of triggers in the context of varying severity of $\mathrm{AF}$ and patient response should be evaluated at multiple institutions to understand the impact on POAF management.

Rhythm control is reserved for patients who cannot tolerate rate control agents or who, after 48 hours, continue to have POAF despite rate control to reduce the risk of thromboembolic events. This recommendation 


\section{Post-operative atrial fibrillation}

Apply IV, $\mathrm{O}_{2}$, monitors. Order ECG, CBC, electrolytes, $\mathrm{Mg}, \mathrm{Ca}, \mathrm{PO}_{4}$

\begin{tabular}{|l|}
\hline Assess stability \\
Unstable \\
If shock (pale, decreased peripheral pulses, hypotension), \\
pulmonary edema, chest pain decreased LOC \\
Synchronized electrocardioversion \\
(I) Arrange for cardiac monitor and intubation equipment; \\
(II) Consider sedation; \\
(III) Synchronized cardioversion at $200 \mathrm{~J}$ monophasic; \\
(IV) Consider echocardiogram to r/o thrombus, and assess for \\
diastolic/systolic dysfunction.
\end{tabular}

\section{Stable}

RATE CONTROL to aim for $\mathrm{HR}<110$.

If decompensated HF or EF $<35 \%$, give amiodarone, NOT beta-blocker or CCB

(I) Otherwise give metoprolol $2 \mathrm{mg}$ IV over $1 \mathrm{~min}$, if favorable response and no adverse events, repeat q5-10 min, initial max IV dose $10 \mathrm{mg}$ total;

(II) If inadequate or no response to $<4 \mathrm{mg}$ metoprolol, give Diltiazem $5 \mathrm{mg}$ IV over $1 \mathrm{~min}$, if favourable response and no adverse events, repeat q10 min PRN, max dose $20 \mathrm{mg}$.

If persistent inadequate ventricular HR response or adverse events, consider:

(I) Digoxin $0.5 \mathrm{mg}$ IV then $0.25 \mathrm{mg}$ IV in $6 \mathrm{~h}$ and if normal

renal function, $0.25 \mathrm{mg}$ IV in $12 \mathrm{~h}$;

(II) Amiodarone $150 \mathrm{mg}$ IV over $10 \mathrm{~min}$ followed by infusion for rate and rhythm control.

RATE MAINTENANCE continue po dosing of agents proven effective in controlling ventricular response HR:

(I) Apo metoprolol, diltiazem, digoxin as indicated.
Assess and manage triggers

Hypoxia?

* Check: lung exam, chest tubes;

* Order: portable CXR;

Electrolyte abnormality?

* Give: $\mathrm{MgSO}_{4} 2 \mathrm{~g}$ IV empirically;

* Replace to maintain $\mathrm{K}>4$ and $\mathrm{Mg}>1 \mathrm{mmol} / \mathrm{L}$;

CAD or CHF?

* Check: HR, JVP;

* Order: CK, TNI, ECG;

Infection?

* Check: wounds, signs of infection;

* Order: CXR Urinalysis PRN;

Bleed or Hypovolemia?

* Check: ins/outs, tubes;

* Give IV fluid bolus PRN.

\begin{tabular}{|c|}
\hline If $48 \mathrm{~h}$ of persistent atrial fibrillation: \\
\hline Assess CHADS2 score [6] \\
\hline CHF [1], HTN [1], Age $>75$ [1], DM [1], History of stroke or TIA [2] \\
\hline
\end{tabular}

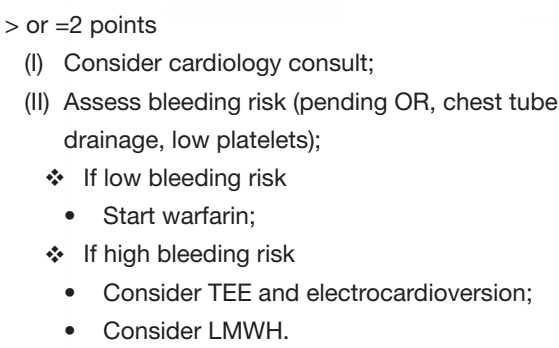

Figure 1 initial individualized management approach to POAF. IV, intravenous; $\mathrm{O}_{2}$, oxygen; ECG, electrocardiogram; CBC, complete blood count; LOC, level of consciousness; HR, heart rate; HF, heart failure; EF, ejection fraction, CHF, congestive heart failure; HTN, hypertension; DM, diabetes mellitus; TIA, transient ischemic attack, CXR, chest X-ray; TEE, trans-esophageal echocardiogram; LMWH, low molecular weight heparin; ASA, acetylsalicylic acid. 
by the AATS is largely based on trials in cardiac surgery and long-term studies in the general population such as the AFFIRM trial which demonstrated rate-control to be superior long-term therapy in patients $>65$ years with AF (8). In the AFFIRM trial, rate control was better tolerated by participants, as demonstrated the 5 -year $37.5 \%$ cross-over rate of patients from rhythm to rate control due to side effects $(\mathrm{P}<0.001)(36)$. Rate control was also associated with significantly fewer hospitalizations, and with less thromboembolic events, major adverse events and mortality although these were not statistically significant. It is unclear to what extent the evidence from this long-term study can be applied to POAF. Evidence specific to thoracic surgery suggests no significant difference between rate and rhythm control, however this is drawn from a single trial of 30 patients comparing amiodarone with diltiazem (35).

The choice of antiarrhythmic therapy for rhythm control should be guided by the patient's comorbidities and the side effect profile of the agent. Procainamide should be avoided in patients with heart failure. Flecainide and propafenone should be avoided in patients with coronary artery disease or structural heart disease. Amiodarone, at high doses, should be used with caution due to concern of pulmonary toxicity (8). Pulmonary toxicity was first noted in a 1994 trial whereby 3 of 11 (27\%) of patients who underwent pneumonectomy and received IV amiodarone for AF developed ARDS (26). However, this number is too small to make any strong recommendations from and multiple trials have since demonstrated no increased risk of pulmonary toxicity with amiodarone even in patients with pneumonectomy. The difference may be that more recent studies have used lower doses. Patients in the 1994 trial patients received cumulative doses of over 2,000 $\mathrm{mg}$ IV whereas more recent trials have only used up to $1,050 \mathrm{mg} \mathrm{IV}$, which has demonstrated to be safe and effective $(27,28)$. As such, careful consideration of patient comorbidities should be considered prior to administering antiarrhythmic therapy and only low doses of IV amiodarone should be used in patients who have undergone major pulmonary resection.

\section{Recommendations}

(I) For patients with new onset POAF who develop acute hemodynamic instability, immediate synchronized electrocardioversion is recommended and consideration of an echocardiogram if POAF is sustained $>48$ hours to detect an atrial thrombus; (II) hemodynamically stable patients with POAF should receive incremental dosing of rate control therapy with continuous cardiac monitoring until a heart rate of $<110 \mathrm{BPM}$ is achieved. If the patient has known decompensated heart failure or ejection fraction $<35 \%$, consider least negatively inotropic agent, namely amiodarone. If there is no decompensated heart failure, initial trial of low trial dose of beta-blocker (e.g., 2-5 mg metoprolol IV push) should be given followed by repeat dosing (every $5 \mathrm{~min}$ ) if beneficial effect witnessed (e.g., lowering HR) and no adverse events (e.g., hypotension, bronchospasm, bradycardia) until desired effect; or if no effect observed on heart rate or on blood pressure, consider CCB (e.g., $5 \mathrm{mg}$ diltiazem IV push), with repeat dosing (q5 min) if beneficial effect and no adverse events to achieve rate control; (III) to augment effect of either beta-blocker or $\mathrm{CCB}$, consider digoxin to further augment effectiveness of rate control and minimize adverse events; (IV) early assessment and correction of underlying POAF triggers may help facilitate early return to sinus rhythm (such as pneumothorax, pneumonia, pulmonary embolism, infection, bleeding or electrolyte abnormalities); (V) cardioversion should be reserved for patients who cannot tolerate rate control agents or who have persistent, poorly controlled POAF despite rate control efforts; (VI) the choice of antiarrhythmic agent should be selected in accordance with the patient's comorbidities and the agents' side effect profile; (VII) further formal evaluations of standardized protocols to predict, prevent and manage POAF are warranted to evaluate impact on incidence, duration and severity of POAF.

\section{Anticoagulation}

Indications for anticoagulation with POAF are controversial and long-term anticoagulation for the prevention of thromboembolic events is rarely required given that POAF is often transient and self-limiting. Rena and colleagues reported that $98 \%$ of patients with POAF after lung resection resolved within 1 day of hospital discharge (39). However, POAF has been shown to be associated with increased risk of ischemic stroke (40). In particular, the few that fail to resolve and remain in non-valvular $\mathrm{AF}$ after 48 hours the risk of stroke is 5 times greater than those in sinus rhythm and therefore early consideration of anticoagulation is recommended. In these patients, anticoagulation therapy should be considered if their individual risk for thromboembolic event according to their risk factors for stroke (i.e., congestive heart failure, hypertension, diabetes, previous thromboembolic event, or peripheral vascular disease) outweighs their 
Table 2 summary of recommendations

Recommendations

(I) Multiple existing predictive models are capable of identifying patients at increased risk for POAF; however clinical application of these risk models first requires external validation, followed by ongoing evaluation and improvement over time;

(II) Prophylactic therapy with beta-blocker agents, amiodarone, calcium-channel blockers (CCB) and magnesium may be considered to reduce the risk of POAF; however providing prophylaxis to all patients is not recommended because the exposure of all patients to the risk of adverse events outweighs the benefit experienced by only a few patients in preventing POAF. The approach of implementing prophylaxis only in high-risk patients only to reduce incidence and severity of POAF is promising yet unproven, and requires further study to evaluate safety and effectiveness;

(III) For patients with new onset POAF who develop acute hemodynamic instability, immediate synchronized electrocardioversion is recommended and consideration for echocardiogram if $>48$ hours to detect possible atrial thrombus;

(IV) Hemodynamically stable patients with POAF should receive incremental dosing of rate control therapy with continuous cardiac monitoring until a heart rate of $<110$ BPM is achieved. If the patient has known decompensated heart failure or ejection fraction $<35 \%$, consider least negatively inotropic agent, namely amiodarone. If no decompensated heart failure, initial trial of low trial dose of beta-blocker (e.g., 2 mg metoprolol IV push) should be given followed by repeat dosing if beneficial effect witnessed (e.g., lowering HR) and no adverse events (e.g., hypotension, bronchospasm, bradycardia) until desired effect; or if no effect observed on heart rate or blood pressure, consider CCB (e.g., 5 mg diltiazem IV push), with repeat dosing if beneficial effect and no adverse events to achieve rate control;

(V) To augment effect of either beta-blocker or CCB, consider digoxin to further augment effectiveness of rate control and minimize adverse events;

(VI) Early assessment and correction of underlying POAF triggers may help facilitate early return to sinus rhythm (such as pneumothorax, pneumonia, pulmonary embolism, infection, bleeding or electrolyte abnormalities);

(VII) Cardioversion should be reserved for patients who cannot tolerate rate control agents or who, after 48 hours, continue to have POAF despite rate control;

(VIII) The choice of antiarrhythmic agent should be selected in accordance with the patient's comorbidities and the agents side effect profile;

(IX) Further formal evaluations of standardized protocols to predict, prevent and manage POAF are warranted to evaluate impact on incidence, duration and severity of POAF;

(X) Antithrombotic therapy to reduce the risk of stroke in patients with persistent AF should be considered if POAF persists $>48$ hours, and should be individualized based on patient's risk factors for thromboembolic event (congestive heart failure, hypertension, diabetes, previous thromboembolic event, peripheral vascular disease) and post-operative bleeding risk

POAF, post-operative atrial fibrillation; CCB, calcium channel blocker; BPM, beats per minute.

risk of post-operative bleeding $(8,41)$. Further detailed recommendations are beyond the scope of this analysis.

\section{Recommendations}

Antithrombotic therapy to reduce the risk of stroke in patients with persistent $\mathrm{AF}$ should be considered if POAF persists $>48$ hours, and should be individualized based on patient's risk factors for thromboembolic event (congestive heart failure, hypertension, diabetes, previous thromboembolic event, peripheral vascular disease) and post-operative bleeding risk.

\section{Conclusions}

This article provides a concise analysis of strategies and practical recommendations (see Table 2) to predict, prevent and manage post-operative AF. Risk factors for POAF are well established and prophylactic therapy has the potential to reduce the incidence of POAF. Validation of prediction models to identify high-risk patients and subsequent trials of prophylaxis in high-risk patients is needed. The mechanisms of AF after surgery are vast and patient response to therapy is variable. As such, management requires close patient monitoring and individualized treatment. Future research should focus on evaluating comprehensive strategies to 
address POAF and continue to standardize initial therapy and in doing so, contribute to the reduction of the overall morbidity of POAF and risk of thromboembolic events associated with this common complication of thoracic surgery.

\section{Acknowledgements}

Thank you to the librarians at The Ottawa Hospital for their assistance with the literature search.

\section{Footnote}

Conflicts of Interest: The authors have no conflicts of interest to declare.

\section{References}

1. Imperatori A, Mariscalco G, Riganti G, et al. Atrial fibrillation after pulmonary lobectomy for lung cancer affects long-term survival in a prospective single-center study. J Cardiothorac Surg 2012;7:4.

2. Amar D, Zhang H, Shi W, et al. Brain natriuretic peptide and risk of atrial fibrillation after thoracic surgery. J Thorac Cardiovasc Surg 2012;144:1249-53.

3. Polanczyk CA, Goldman L, Marcantonio ER, et al. Supraventricular arrhythmia in patients having noncardiac surgery: clinical correlates and effect on length of stay. Ann Intern Med 1998;129:279-85.

4. Devereaux PJ, Yang H, Yusuf S, et al. Effects of extendedrelease metoprolol succinate in patients undergoing noncardiac surgery (POISE trial): a randomised controlled trial. Lancet 2008;371:1839-47.

5. Camm AJ, Lip GY, De Caterina R, et al. 2012 focused update of the ESC Guidelines for the management of atrial fibrillation: an update of the 2010 ESC Guidelines for the management of atrial fibrillation--developed with the special contribution of the European Heart Rhythm Association. Europace 2012;14:1385-413.

6. Rao VP, Addae-Boateng E, Barua A, et al. Age and neo-adjuvant chemotherapy increase the risk of atrial fibrillation following oesophagectomy. Eur J Cardiothorac Surg 2012;42:438-43.

7. Cardinale D, Sandri MT, Colombo A, et al. Prevention of Atrial Fibrillation in High-risk Patients Undergoing Lung Cancer Surgery: The PRESAGE Trial. Ann Surg 2016;264:244-51.

8. Frendl G, Sodickson AC, Chung MK, et al. 2014
AATS guidelines for the prevention and management of perioperative atrial fibrillation and flutter for thoracic surgical procedures. J Thorac Cardiovasc Surg 2014;148:e153-93.

9. Lewis T. Report cxix. auricular fibrillation: a common clinical condition. Br Med J 1909;2:1528.

10. Munger TM, Wu LQ, Shen WK. Atrial fibrillation. J Biomed Res 2014;28:1-17.

11. Elahi MM, Flatman S, Matata BM. Tracing the origins of postoperative atrial fibrillation: the concept of oxidative stress-mediated myocardial injury phenomenon. Eur J Cardiovasc Prev Rehabil 2008;15:735-41.

12. Gaudino M, Andreotti F, Zamparelli R, et al. The -174G/ C interleukin-6 polymorphism influences postoperative interleukin-6 levels and postoperative atrial fibrillation. Is atrial fibrillation an inflammatory complication? Circulation 2003;108 Suppl 1:II195-9.

13. Maesen B, Nijs J, Maessen J, et al. Post-operative atrial fibrillation: a maze of mechanisms. Europace 2012;14:159-74.

14. Onaitis M, D'Amico T, Zhao Y, et al. Risk factors for atrial fibrillation after lung cancer surgery: analysis of the Society of Thoracic Surgeons general thoracic surgery database. Ann Thorac Surg 2010;90:368-74.

15. Kotova S, Wang M, Lothrop K, et al. CHADS2 Score Predicts Postoperative Atrial Fibrillation in Patients Undergoing Elective Pulmonary Lobectomy. Ann Thorac Surg 2017;103:1566-72.

16. Cardinale D, Colombo A, Sandri MT, et al. Increased perioperative $\mathrm{N}$-terminal pro-B-type natriuretic peptide levels predict atrial fibrillation after thoracic surgery for lung cancer. Circulation 2007;115:1339-44.

17. Simmers D, Potgieter D, Ryan L, et al. The use of preoperative B-type natriuretic peptide as a predictor of atrial fibrillation after thoracic surgery: systematic review and meta-analysis. J Cardiothorac Vasc Anesth 2015;29:389-95.

18. Amar D, Zhang H, Miodownik S, et al. Competing autonomic mechanisms precede the onset of postoperative atrial fibrillation. J Am Coll Cardiol 2003;42:1262-8.

19. Passman RS, Gingold DS, Amar D. Prediction Rule for Atrial Fibrillation After Major Noncardiac ThoracicSurgery. Ann Thorac Surg 2005;79:1698-703.

20. Vaporciyan AA, Correa AM, Rice DC, et al. Risk factors associated with atrial fibrillation after noncardiac thoracic surgery: analysis of 2588 patients. J Thorac Cardiovasc Surg 2004;127:779-86.

21. Fernando HC, Jaklitsch MT, Walsh GL, et al. The Society 
of Thoracic Surgeons practice guideline on the prophylaxis and management of atrial fibrillation associated with general thoracic surgery: executive summary. Ann Thorac Surg 2011;92:1144-52.

22. Tada H, Sticherling C, Chough SP, et al. Gender and age differences in induced atrial fibrillation. Am J Cardiol 2001;88:436-8.

23. Zhao BC, Huang TY, Deng QW, et al. Prophylaxis Against Atrial Fibrillation After General Thoracic Surgery: Trial Sequential Analysis and Network Meta-Analysis. Chest 2017;151:149-59.

24. Talati R, Reinhart KM, White CM, et al. Outcomes of perioperative beta-blockade in patients undergoing noncardiac surgery: a meta-analysis. Ann Pharmacother 2009;43:1181-8.

25. Khalil MA, Al-Agaty AE, Ali WG, et al. A comparative study between amiodarone and magnesium sulfate as antiarrhythmic agents for prophylaxis against atrial fibrillation following lobectomy. J Anesth 2013;27:56-61.

26. Van Mieghem W, Coolen L, Malysse I, et al. Amiodarone and the development of ARDS after lung surgery. Chest 1994;105:1642-5.

27. Kolokotroni SM, Toufektzian L, Harling L, et al. In patients undergoing lung resection is it safe to administer amiodarone either as prophylaxis or treatment of atrial fibrillation? Interact Cardiovasc Thorac Surg 2017;24:783-8.

28. Zhang L, Gao S. Systematic Review and Meta-analysis of Atrial Fibrillation Prophylaxis After Lung Surgery. J Cardiovasc Pharmacol 2016;67:351-7.

29. January CT, Wann LS, Alpert JS, et al. 2014 AHA/ ACC/HRS guideline for the management of patients with atrial fibrillation: a report of the American College of Cardiology/American Heart Association Task Force on practice guidelines and the Heart Rhythm Society. Circulation 2014;130:e199-267.

30. Personett HA, Smoot DL, Stollings JL, et al. Intravenous metoprolol versus diltiazem for rate control in noncardiac, nonthoracic postoperative atrial fibrillation. Ann Pharmacother 2014;48:314-9.

31. Balser JR, Martinez EA, Winters BD, et al. $\gamma$-Adrenergic

Cite this article as: Smith $\mathrm{H}$, Yeung C, Gowing S, Sadek M, Maziak D, Gilbert S, Shamji F, Villeneuve P, Sundaresan R, Seely A. A review and analysis of strategies for prediction, prevention and management of post-operative atrial fibrillation after non-cardiac thoracic surgery. J Thorac Dis 2018;10(Suppl 32):S3799-S3808. doi: 10.21037/jtd.2018.09.144
Blockade Accelerates Conversion of Postoperative Supraventricular Tachyarrhythmias. Anesthesiology 1998;89:1052-9.

32. Mooss AN, Wurdeman RL, Mohiuddin SM, et al. Esmolol versus diltiazem in the treatment of postoperative atrial fibrillation/atrial flutter after open heart surgery. Am Heart J 2000;140:176-80.

33. Martindale JL, deSouza IS, Silverberg M, et al. $\beta$-Blockers versus calcium channel blockers for acute rate control of atrial fibrillation with rapid ventricular response: a systematic review. Eur J Emerg Med 2015;22:150-4.

34. Fromm C, Suau SJ, Cohen V, et al. Diltiazem vs. Metoprolol in the Management of Atrial Fibrillation or Flutter with Rapid Ventricular Rate in the Emergency Department. J Emerg Med 2015;49:175-82.

35. Bobbio A, Caporale D, Internullo E, et al. Postoperative outcome of patients undergoing lung resection presenting with new-onset atrial fibrillation managed by amiodarone or diltiazem. Eur J Cardiothorac Surg 2007;31:70-4.

36. Olshansky B, Rosenfeld LE, Warner AL, et al. The Atrial Fibrillation Follow-up Investigation of Rhythm Management (AFFIRM) study: approaches to control rate in atrial fibrillation. J Am Coll Cardiol 2004;43:1201-8.

37. Amar D, Piening D, Zhang H, et al. Clinical prediction model for postoperative atrial fibrillation (POAF). J Cardiothorac Vasc Anesth 2016;30:S19.

38. Walsh SR, Thomas C, Manohar S, et al. Early management of atrial fibrillation in general surgical inpatients. Int J Surg 2006;4:115-7.

39. Rena O, Papalia E, Oliaro A, et al. Supraventricular arrhythmias after resection surgery of the lung. Eur J Cardiothorac Surg 2001;20:688-93.

40. Gialdini G, Nearing K, Bhave PD, et al. Perioperative atrial fibrillation and the long-term risk of ischemic stroke. JAMA 2014;312:616-22.

41. Kannel WB, Wolf PA, Benjamin EJ, et al. Prevalence, incidence, prognosis, and predisposing conditions for atrial fibrillation: population-based estimates. Am J Cardiol 1998;82:2N-9N. 